\title{
Phase diagram of the one-dimensional extended attractive Hubbard model for large nearest-neighbor repulsion
}

\author{
A. A. Aligia \\ Comisión Nacional de Energía Atómica, \\ Centro Atómico Bariloche and Instituto Balseiro, 8400 S.C. de Bariloche, \\ Argentina. \\ (Received October 5, 2018)
}

\begin{abstract}
We consider the extended Hubbard model with attractive on-site interaction $U$ and nearest-neighbor repulsions $V$. We construct an effective Hamiltonian $H_{\text {eff }}$ for hopping $t<<V$ and arbitrary $U<0$. Retaining the most important terms, $H_{\text {eff }}$ can be mapped onto two $X X Z$ models, solved by the Bethe ansatz. The quantum phase diagram shows two Luttinger liquid phases and a region of phase separation between them. For density $n<0.422$ and $U<-4$, singlet superconducting correlations dominate at large distances. For some parameters, the results are in qualitative agreement with experiments in $\mathrm{Ba}_{1-x} \mathrm{~K}_{x} \mathrm{BiO}_{3}$.
\end{abstract}

\section{INTRODUCTION}

$\mathrm{BaPb}_{1-x} \mathrm{Bi}_{x} \mathrm{O}_{3}$ and $\mathrm{Ba}_{1-x} \mathrm{~K}_{x} \mathrm{BiO}_{3}$ are superconduc tors with transition temperatures $T_{c}$ near $13 \mathrm{~KB}$ and $30 \mathrm{~K}$ respectively. In spite of the fact that these systems do not contain $\mathrm{Cu}$ and are non-magneticB, they have important similarities with the cuprate superconductors, like the perovskite structure, relatively high $T_{c}$ and low density of states at the Fermi level3. While the electron-phonon interaction is very important in these materials, as shown by inelastic neutron measurements 1 , and the existence of displacements of $\mathrm{O}$ atoms and lattice distortions 5 , several other experiments suggest that the pairing mechanism is, at least in part, of electropic nature3. Excitons combined with $\mathrm{O}$ displacements tractive interaction $U$ based on non-linear screening 1012 , have been proposed as the origin of superconductivity in doped $\mathrm{BaBiO}_{3}$. In these approaches, repulsive interactions at finite distances play an important role, what is consistent with poor screening of Coulomb interactions by the low density of carriers.

As first shown by Rice and Sneddon 12 , treating the displacements $d$ of $\mathrm{O}$ ions in the direction of their nearestneighbor $\mathrm{Bi}$ ions in the antiadiabatic approximation, leads to a decrease in $U$ by $z g^{2} / K$, and an increase of the repulsion $V$ between nearest $\mathrm{Bi}$ atoms by $g^{2} / K$, where $z$ is the coordination number, $g$ the electron-phonon interaction, and $K$ the second derivative of the elastic energy with respect to $d$. This leads naturally to the extended Hubbard model for the description of doped $\mathrm{BaBiO}_{3}$, and to superconductivity carried by bipolarons (a pair of carriers at $\mathrm{Bi}$ sites accompanied by $\mathrm{O}$ displacements) 2 , 13 . While the excitonic mechanism involves partial occupation of $\mathrm{O}$ states by holes 8 , in agreement with optical experiments 1416 , it is still possible that this oneband model describes the low-energy physics (as in the cuprates 17). This is certainly the case in the purely elec- tronic model proposed by Varma, in which non-linear screening not only reduces the bare atomic $U \sim 11 \mathrm{eV}$, but renders it negative 1 .

In recent years, the phase diagram of the extended Hubbard model in one dimension has been studied by numerical techniques, with particular emphasis in the frar. ter filled case (number of particles per site $n=1 / 2$ ) 18 24. For $n=1 / 2$ or $n=2 / 3, V>5$ and small negative $U$, calculations of the correlation exponent $K_{\rho}$ in systems of up to $L=16$ sites, predict a Luttinger liquid phase with dominant superconducting correlations at large distances 19., ed. However, from an analysis based on the infinite $V$ limit and the extrapolation of the spin gap, Penc and Mila suggested that the results of $K_{\rho}$ were affected by finite-size effects, and the system would not be a Luttinger liquid in that region 19 . For $n=1 / 2$, perturbative arguments around the infinite $V$ limit (developed in more detail here) suggested that this region corresponds to phase separation 22. Mpnte Carlo studies in systems with $L \sim 64$ lattice sites 23 have shown that the region of phase separation in the model extends to much lower values of $V$ than those obtained for $L \leq 16$, leaving practically no place for a Luttinger liquid phase with dominant superconducting correlations for large $V$. The extent of this phase remains unclear. Furthermore, due to the above mentioned finite-size effects, and technical problems with different Monte Carlo methods23, the region of small $|U|$ and $V>8 t$, where $t$ is the hopping, is practically unaccessible to the present available numerical methods. This region is also out of the range of applicability of the continuum-limit field theory (also called $g$-ology), which has been pplied to the extended Hubbard and related models 25 es.

In this work, we study the one-dimensional attractive Hubbard model in the limit $V>>t$. Extending previous work 19 , we derive an effective low-energy Hamiltonian including terms up to second order in $t$. Under certain approximations, which are not essential for sufficiently 
large $V$, the effective Hamiltonian is mapped into two $X X Z$ models, representing the movements of particles in singly and doubly occupied sites respectively. From the Bethe ansatz solution of these models, the phase diagram in the thermodynamic limit $L \rightarrow \infty$ as a function of $n, U / t<0$ and large $V / t$ is obtained. In the next Section, we explain the model and the mapping procedures. The results are shown in Section III. Section IV contains the conclusions and a discussion of the possible relation between our results and the phase diagram measured in $\mathrm{Ba}_{1-x} \mathrm{~K}_{x} \mathrm{BiO}_{3} \mathrm{l}$.

\section{MODEL AND EFFECTIVE HAMILTONIAN}

The one-dimensional extended Hubbard Hamiltonian in standard notation is:

$H=-t \sum_{i \sigma}\left(c_{i+1 \sigma}^{\dagger} c_{i \sigma}+\right.$ H.c. $)+U \sum_{i} n_{i \uparrow} n_{i \downarrow}+V \sum_{i} n_{i+1} n_{i}$,

and we consider the case $U<0, V>>t$. In this limit, there are no nearest-neighbor occupied sites in the low-energy subspace. The terms in Eq. (11) which mix states of this subspace with states with non-zero occupancy at nearest-neighbor sites, can be eliminated through a standard canonical transformation, as described in Ref.69. This procedure originates terms of order $t^{2} / V$ or smaller in the low-energy subspace. The resulting effective Hamiltonian within this subspace (a projector $P=\prod_{i \sigma \sigma^{\prime}}\left(1-n_{i \sigma} n_{i+1 \sigma^{\prime}}\right)$ is implicit) can be written in the form:

$$
H_{e f f}=H_{s}+H_{d}+H_{s d}
$$

where $H_{s}$ involves only singly occupied sites:

$$
\begin{aligned}
H_{s}= & -t \sum_{i \sigma}\left(c_{i+1 \sigma}^{\dagger} c_{i \sigma}+\text { H.c. }\right)\left(1-n_{i \bar{\sigma}}\right)\left(1-n_{i+1 \bar{\sigma}}\right) \\
& +V_{s} \sum_{i \sigma \sigma^{\prime}} n_{i \sigma} n_{i+2 \sigma^{\prime}}\left(1-n_{i \bar{\sigma}}\right)\left(1-n_{i+2 \bar{\sigma}}\right) \\
& +V_{s} \sum_{i \sigma \sigma^{\prime}}\left(c_{i+3 \sigma}^{\dagger} c_{i+2 \sigma} c_{i+1 \sigma^{\prime}}^{\dagger} c_{i \sigma^{\prime}}+\text { H.c. }\right),
\end{aligned}
$$

with $V_{s}=-t^{2} / V$. The second term is an interaction between second nearest neighbors, and the third term displaces two next-nearest-neighbor particles one lattice parameter.

The second term in Eq. (2) can be described entirely in terms of the operators $d_{i}^{\dagger}=c_{i \uparrow}^{\dagger} c_{i \downarrow}^{\dagger}$, which create "doublons" at each site:

$$
\begin{aligned}
H_{d}= & \Delta_{d} \sum_{i} d_{i}^{\dagger} d_{i}-t_{d} \sum_{i \sigma}\left(d_{i+1}^{\dagger} d_{i}+\text { H.c. }\right) \\
& +V_{d} \sum_{i} d_{i+2}^{\dagger} d_{i+2} d_{i}^{\dagger} d_{i},
\end{aligned}
$$

where $\Delta_{d}=U-4 t^{2} /(V-U), t_{d}=2 t^{2} /(V-U)$, and $V_{d}=4 t^{2}[1 /(V-U)-1 /(3 V-U)]$ are the effective onsite energy, nearest-neighbor hopping, and next-nearestneighbor repulsion respectively, for doublons.

The last term in Eq. (2) describes interactions between singly and doubly occupied sites:

$$
\begin{aligned}
H_{s d}= & M_{1} \sum_{i}\left[\left(c_{i+1 \uparrow}^{\dagger} c_{i-1 \downarrow}^{\dagger}+c_{i-1 \uparrow}^{\dagger} c_{i+1 \downarrow}^{\dagger}\right)\right. \\
& \left.\times\left(1-n_{i-1}\right)\left(1-n_{i+1}\right)\left(d_{i-1}+2 d_{i}+d_{i+1}\right)+\text { H.c. }\right] \\
& +M_{2} \sum_{i \sigma}\left(c_{i \sigma}^{\dagger} d_{i+2}^{\dagger} d_{i} c_{i+2 \sigma}+\text { H.c. }\right) \\
& +V_{s d} \sum_{i \sigma \delta= \pm 2} n_{i \sigma}\left(1-n_{i \bar{\sigma}}\right) d_{i+\delta}^{\dagger} d_{i+\delta} .
\end{aligned}
$$

Here $M_{1}=-t^{2}[1 / V+1 /(V-U)] / 2$ describes annihilation of a doublon with creation of two particles at empty sites, and the Hermitian conjugate process, while $M_{2}=$ $t^{2} /(2 V-U)\left(V_{s d}=t^{2}[2 /(V-U)-2 /(2 V-U)-1 /(2 V])\right.$ corresponds to interchange (interaction) of a doublon and a particle at a singly occupied next-nearest-neighbor site.

For $V=+\infty$, all terms of $H_{\text {eff }}$ vanish except the first term of Eq. (3) and the first term of Eq. (4), and $H_{\text {eff }}$ can be solved exactly 19 . In this limit, for $U>-4 t$ and sufficiently small density $n$, the system has no doubly occupied sites and is described by $H_{s}$. Instead, for $U<$ $-4 t, n \leq 1$, the ground state of $H_{\text {eff }}$ is the same as that of $H_{d}$ and has no singly occupied sites. For other values of $U$ and $n \leq 1$, the system phase separates into the phases just described, and the limits of the region of phase separation (PS) can be obtained using the Maxwell construction (finding the common tangent to the curves $E_{s}(n)$ and $E_{d}(n)$, where $E_{\alpha}(n)$ is the ground state of $H_{\alpha}$ at density $n$ ).

For the sake of clarity we call "metallic" (M) the phase without double occupancy (ground state of $H_{s}$ ), and "bipolaronic" (BP), the phase described by the ground state of $H_{d}$, although the negative $U$ is not necessarily related with atomic displacements in a real system. For finite but large $V$, the energy cost for constructing a uniform phase with both, singly and doubly occupied sites is high in comparison with $M_{1}, M_{2}$ and $V_{s d}$. Thus, in the ground state, $H_{s d}$ can only act in the PS region, at the boundary between $\mathrm{M}$ and $\mathrm{BP}$ phases, and is irrelevant in the thermodynamic limit. Our main approximation is the neglect of $H_{s d}$. This should be correct as long as the energy gain of $H_{s}$ in the PS region $(\sim t)$ is larger than the terms of $H_{s d}\left(\sim t^{2} / V\right)$. We also neglect the last term of Eq. (3). This term vanishes at the extreme densities of the $\mathrm{M}$ phase $(n=0$ and $n=1 / 2)$. The ratio of its expectation value with respect to the expectation value of the first term of Eq. (3) can be estimated by perturbation theory:

$$
r=\frac{t}{V}\left[n_{s} \cos \left(\pi n_{s}\right)-\frac{1}{\pi} \sin \left(\pi n_{s}\right)\right],
$$

where $n_{s}=n /(1-n)$. The remaining terms of $H_{s}$, 
and the whole of $H_{d}$ can both be mapped into a spinless fermion model $H_{\alpha}^{s f}(\alpha=s$ or $d)$ :

$$
\begin{aligned}
H_{\alpha}^{s f}= & \Delta_{\alpha} \sum_{i} f_{i}^{\dagger} f_{i}-t_{\alpha} \sum_{i \sigma}\left(f_{i+1}^{\dagger} f_{i}+\text { H.c. }\right) \\
& +V_{\alpha} f_{i}^{\dagger} f_{i} f_{i+1}^{\dagger} f_{i+1},
\end{aligned}
$$

with $\Delta_{s}=0$. When $\alpha=s$, a site occupied by a fermion $f_{i}^{\dagger}$ corresponds to a single occupied site and an empty site at the right of it, as explained in detail by Penc and Mila 19 (a similar mapping was also used in a model for oxygen ordering in $\mathrm{YBa}_{2} \mathrm{Cu}_{3} \mathrm{O}_{6+x} 29$ ). Then, for $L$ sites and $N$ particles in $H_{s}$, the corresponding number of sites and particles in $H_{s}^{s f}$ are $L_{s}=L-N, N_{s}=N$. Then, the energy per site $e_{s}(n)$ of $H_{s}$ for density $n$ is related to the corresponding quantity $e_{s}^{s f}\left(n_{s}\right)$ of $H_{s}^{s f}$ by:

$$
e_{s}(n)=\frac{E_{s}}{L}=\frac{L_{s}}{L} \frac{E_{s}^{s f}}{L_{s}}=(1-n) e_{s}^{s f}\left(n_{s}\right) .
$$

Similarly, $H_{d}$ can be cast into the form of Eq. (7), mapping a doubly occupied site and an empty site at the right of it into a single site occupied by a fermion. The mapping of the different physical quantities is the same as that used before to find the correlation exponent $K_{\rho}$ in a generalized $t-J$ model with very large three-site term Bd. 31. The number of sites and fermions in $H_{d}^{s f}$ are $L_{d}=L-N / 2, N_{d}=N / 2$. The energy per site of $H_{d}$ is given in terms of that of $H_{d}^{s f}$ by:

$$
e_{d}(n)=\left(1-\frac{n}{2}\right) e_{d}^{s f}\left(n_{d}\right) ; n_{d}=\frac{n}{2-n} .
$$

To calculate $K_{\rho}$, we also need the mapping of the velocity 30 :

$$
v_{d}=\frac{L}{L_{d}} v_{d}^{s f}=\frac{2}{2-n} v_{d}^{s f},
$$

and/or the Drude weight:

$$
D_{d}=\frac{L}{2} \frac{\partial^{2} E_{d}(\Phi)}{\partial \Phi^{2}}=4 \frac{L_{d}}{L} \frac{\partial^{2} E_{d}^{s f}\left(\Phi_{s f}\right)}{\partial \Phi_{s f}^{2}}=2(2-n) D_{d}^{s f} .
$$

Here $E_{d}(\Phi)\left(E_{d}^{s f}\left(\Phi_{s f}\right)\right)$ is the energy of a ring described by $H_{d}\left(H_{d}^{s f}\right)$ threaded by a flur $\Phi\left(\Phi_{s f}\right)$. The correlation exponent can be calculated as 31 :

$$
K_{\rho}=\frac{\pi v_{d}}{2 \partial^{2} e_{d} / \partial n^{2}}=\frac{\pi D_{d}}{v_{d}}=\pi\left(\frac{D_{d}}{2 \partial^{2} e_{d} / \partial n^{2}}\right)^{1 / 2} .
$$

Similar expressions give $K_{\rho}$ for $H_{s}$, but we do not give them, since in the M phase always $K_{\rho}<1$, and we are interested in the region $K_{\rho}>1$, for which superconducting correlations dominate at large distances.

Using a Jordan-Wigner transformation, $H_{\alpha}^{s f}$ is transformed into an equivalent $X X Z$ model with $L_{\alpha}$ sites and $M_{\alpha}$ spins down:

$$
\begin{aligned}
H_{\alpha}^{s f} \equiv & H_{\alpha}^{X X Z}=2 t_{\alpha} \sum_{i}\left(S_{i}^{x} S_{i+1}^{x}+S_{i}^{y} S_{i+1}^{y}\right)+V_{\alpha} \sum_{i} S_{i}^{z} S_{i+1}^{z} \\
& +\Delta_{\alpha} N_{\alpha}+V_{\alpha}\left(N_{\alpha}-L_{\alpha} / 4\right) .
\end{aligned}
$$

We have calculated the energy $e_{\alpha}^{s f}\left(n_{\alpha}\right)$ solving numerically the integral equations of the exact Bethe ansatz solution of Eq. (13) in the thermodynamic limit32. To obtain $K_{\rho}$, we have calculated the excitation energies for two small momenta, solving mumerically the corresponding Bethe ansatz equations 33 . This allowed us to extract $v_{d}^{s f}\left(n_{d}\right)$. From it, the numerical second derivative of $e_{d}(n)$, Eqs. (9), (10) and the first Eq. (12), $K_{\rho}$ was calculated for $n_{d} \neq 1 / 2$. For $n_{d}=1 / 2$, we had technical problems in the calculation of $v^{s f}\left(n_{d}\right)$, but fortunately, analytical expressions are known 34 [ 35 :

$$
\begin{aligned}
v_{d}^{s f} & =\frac{\pi t \sin \mu}{\mu} ; D_{d}^{s f}=\frac{v_{d}^{s f}}{4(\pi-\mu)} ; \\
\mu & =\arccos (V / 2 t) .
\end{aligned}
$$

Using Eqs. (10), (11), 12) and (14), one has for $n=2 / 3$ $\left(n_{d}=1 / 2\right)$ :

$$
K_{\rho}(2 / 3)=\frac{4}{9(1-\mu / \pi)} .
$$

For $V_{\alpha}=0, H_{\alpha}^{s f}$ can be solved trivially 30 and Eqs.(9), (10), (11), (12) lead to another analytical result for $V_{d}=$ $0:$

$$
K_{\rho}(2 / 3)=\frac{(2-n)^{2}}{2} .
$$

Since always $V_{d} / t_{d}>0$, and $K_{\rho}$ decreases with increasing $V_{d}$, Eq. (16) implies that for large $V$, no phase with dominant superconducting correlations exists for $n \geq 2-$ $\sqrt{2} \simeq 0.59$.

\section{BETHE ANSATZ RESULTS}

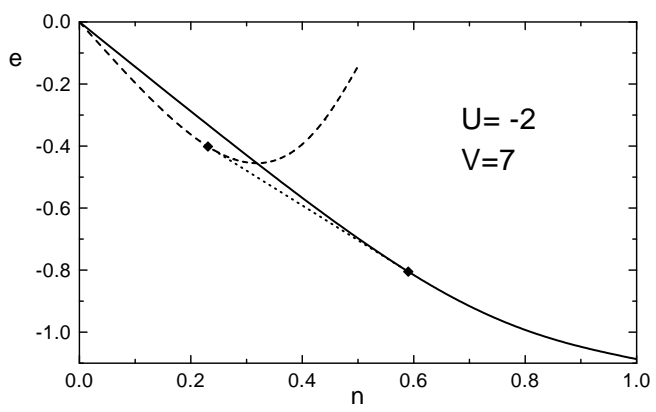

FIG. 1. Ground state energy of the phase with singly occupied sites (dashed line) and doubly occupied sites (full line) as a function of density. The dotted line is the Maxwell construction (see text). 
In Fig. 1 we show the energy per site of $H_{s}\left(e_{s}(n)\right)$ with the last term neglected, and that of $H_{d}\left(e_{d}(n)\right)$, as a function of density $n$ for $U=-2$ and $V=7$. We take $t=1$ as the unit of energy. The dotted line represents the energy for average composition $n$, of an inhomogeneous (phase separated) mixture of the "metallic" (M) phase described by $H_{s}$ for density $n_{1}=0.2307$ and the "bipolaron" (BP) phase (ground state of $H_{d}$ ) for density $n_{2}=0.5901$. These compositions $n_{i}$, represented by diamonds in Fig. 1, are obtained finding the common tangent to both curves $e_{s}(n)$ and $e_{d}(n)$ (Maxwell construction). Between them, the energy of the phase separated phase is lower than both $e_{s}(n)$ and $e_{d}(n)$. The energy $e_{s}(n)$ is dominated by the first term of Eq. (3), which already exists for $V=+\infty$. The effect of finite large $V$ is small (except near $n=1 / 2$ for which the first term of Eq. (3) vanishes), and does not change $n_{1}$ significantly. Instead, the effect of a finite large $V$ on $n_{2}$ is dramatic, reducing it from $n_{2}=1$ to $n_{2}<0.6$. This is because for $V=+\infty, e_{d}(n)$ is always a straight line (extending from $e_{d}(0)=0$ to $e_{d}(1)=-1$ if $\left.U=-2\right)$. The second and third term of $H_{d}$ (Eq.(化)), taken into account exactly, are responsible of the curvature of $e_{d}(n)$ and the shift of $n_{2}$ from 1. This is the main effect of finite large $V$.

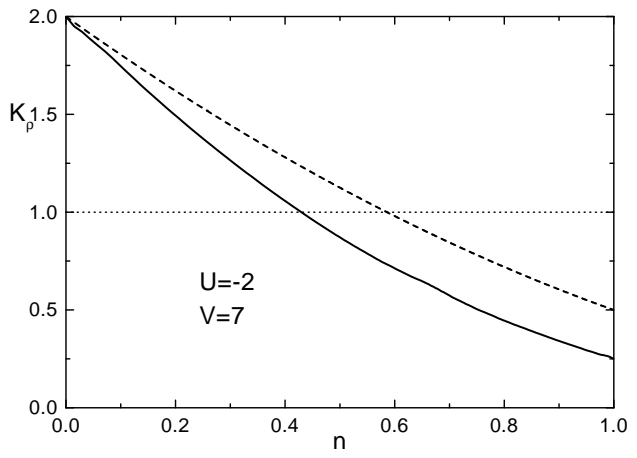

FIG. 2. Correlation exponent $K_{\rho}$ as a function of density in the "bipolaron" (BP) phase for $U=-2$ and $V=7$ (full line). The dashed line is the result in absence of the effective nearest-neighbor repulsion between doubly occupied sites $\left(V_{d}=0\right)$.

Another important effect of a finite $V$ is that the pairs acquire mobility and for low densities, superconducting correlations dominate at large distances $\left(K_{\rho}>1\right)$ in the BP phase. In Fig. 2 we represent $K_{\rho}(n)$ in this phase for the same parameters of Fig. 1. Also shown is the analytical result Eq. (16), which is an upper bound of $K_{\rho}$ for any values of $U<0$ and $V>>t$. For $V \rightarrow+\infty$, we obtain a critical density $n_{c}=0.422$ for which $K_{\rho}\left(n_{c}\right)=1$. For $n<n_{c}$ and arbitrary values of $U<0$ and $V>>t$, the exponent $K_{\rho}(n)>1$. Thus, $n_{c}$ lies in the interval $(0.422,2-\sqrt{2})$. For $-5 \leq U \leq 0$ and $5 \leq V \leq 20$, we find that $n_{c}<0.45$. Also, $n_{c}$ depends very weakly on $U$ and $V$ within the studied range of parameters, decreasing with increasing repulsions. For $U=-2, V=7$ (as in Figs. 1 and 2), $n_{c}=0.429$. These results disagree with thoseptained by numerical diagonalization of small systems 19.20 , which obtained $K_{\rho}>1$ for small values of $|U|$, large $V$ and $n=1 / 2, n=2 / 3$, but agree with the statement that the system might not be a Luttinger liquid in that regiont 9 , and with recent Monte Carlo results in larger systems 23 . These results and the ones shown below indicate that there is phase separation (PS) in that region. We believe that the reason of the artificially large values of $K_{\rho}$ in the above mentioned numerical results is that they were calculated using the first Eq. (12) with $\partial^{2} e / \partial n^{2}=1 /\left(\kappa n^{2}\right)$ determined numerically from the energy for $N, N-2$ and $N+2$ particles with $N / L=n$. In phase separated regions (dashed line in Fig. 1), the compressibility $\kappa$ diverges in the thermodynamic limit, but in small systems, $\kappa$ can be large and positive due to finite-size effects, leading to very large values of $K_{\rho}$, while in fact the system is not a Luttinger liquid. This effect was present in numerical studies of the one-dimensional $t-J$ model with correlated hopping 36 .

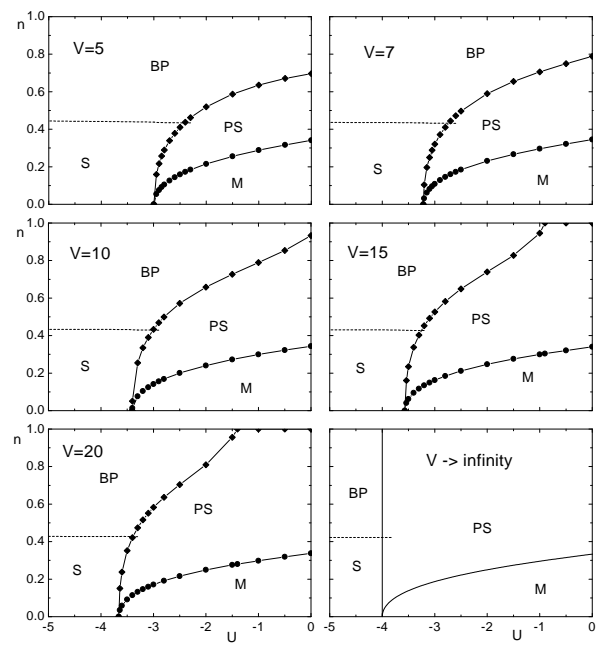

FIG. 3. Phase diagram of the model in the density- $U$ plane, for different values of $V$. The phase without doubly occupied sites is called "metallic" $(\mathrm{M})$, while the phase with no singly occupied sites is denoted as "bipolaronic" (BP), and if $K_{\rho}>1$ we call it "superconducting" (S). The region of phase separation is labeled PS. 
In Fig. 3 we show the phase diagram of the model, determined using the Maxwell construction (as in Fig. 1) for different values of $U$ and $V$ (points indicated with solid symbols in Fig. 3), and searching the critical densities $n_{c}$ for which $K_{\rho}\left(n_{c}\right)=1$. The phase diagram for $V \rightarrow+\infty$ was already known, except for the boundary at $n=0.422$, which separates the BP phase with $K_{\rho}<1$ (in which charge correlation functions are the dominant ones at large distances) from the "superconducting" (S) phase with $K_{\rho}>1$. The most noticeable effect of a finite $V$, already present for $V$ as large as $20 t$ is the change in the boundary between the BP and PS regions, as a consequence of the above mentioned curvature of $e_{d}(n)$ for $V<+\infty$. Also, as $V$ decreases, the region $\mathrm{S}$ with $K_{\rho}>1$ increases, moving to larger values of $U$ and to slightly larger densities. Finally, the PS region is reduced.

The case $V=5$ is probably beyond the quantitative validity of our large $V$ appreximation. Monte Carlo results for $V=8$ (Fig. 14 of Ref.23) are in qualitative agreement with our results. Quantitatively, Clay et al. obtain that for $U=0$, phase separation begins at $n_{1} \sim 0.5$, while for $V=7$ we obtain $n_{1}=0.345$. This difference might be due to the effect of terms of order $t^{m}$ with $m>2$ or the third term of $H_{s}$ (Eq. (3)), which we have neglected. Terms of order $t^{4}$ reduce $t_{d}$ and increase $n_{1}$. The limit between $\mathrm{S}$ and $\mathrm{M}$ phases at low densities is affected neither by $H_{s d}$ (Eq. (5)) nor by the third term of $H_{s}$, but might be changed slightly by terms of order $t^{4}$.

\section{SUMMARY AND DISCUSSION}

Generalizing a previous approach 19 , we have constructed an effective Hamiltonian $H_{\text {eff }}$ for the extended Hubbard model when $V>>t$ and $U \leq 0$. This permits to study the region $V>8 t$, which is outside the region of validity of weak-coupling approaches 25 while numerical diagonalization of small systems 19.20,22 display important finite-size effects, as discussed in Section III, and Monte Carlo calculations have some technical problems. The effective Hamiltonian can be divided in three parts: $H_{s}, H_{d}$ and $H_{s d}$. The latter is irrelevant for sufficiently large $V$, and $H_{s}\left(H_{d}\right)$ acts on a phase in which all particles move in singly (doubly) occupied sites. $H_{d}$ and the most important terms of $H_{s}$ were mapped into a Bethe ansatz exactly solvable model, which allows to obtain the energy and correlation exponent $K_{\rho}$ in the thermodynamic limit. From this information we have constructed the phase diagram. We obtain a region for low and intermediate densities and sufficiently negative $U$, in which the system behaves as a Luttinger liquid with dominant superconducting correlations at large distances, for any finite $V>>t$. The existence of this phase and the main changes in the phase diagram with respect to the $V=+\infty$ limit, are due to the dynamics of doubly occupied sites in $H_{d}$, controled by terms of order $t^{2} /(V-U)$. The most noticeable effect of a finite $V$ for moderate val- ues of $|U|(U>-4)$, is that the upper density of the phase separation (PS) region is reduced from $n=1$ to much lower values. Instead, the larger density for which superconducting correlations dominate at large distances remains near $n=0.42$. Thus, the place left by the PS region is mostly occupied by the BP ("bipolaronic" "normal") phase rather than by the S ("superconducting") phase. Nevertheless, as $V$ decreases from very large values to $V \sim 7$, the upper value of $U$ for which the $\mathrm{S}$ phase exists increases from -4 to $\sim-3$.

The extended Hubbard model with attractive $U$ can be justified in different ways, as a model for doped $\mathrm{BaBiO}_{3}$, as discussed in Section I. In spite of the different dimensionality of the real compound, one can discuss qualitatively the physics expected from the phase diagram (Fig. $3)$. $\mathrm{BaBiO}_{3}$ has one electron per site $(n=1)$, and the ground state of the model is a charge density wave, in which nearest-neighbor sites are not equivalent, as experimentally observed 0 . For sufficiently negative $U$, as $n$ decreases (corresponding to partial replacement of $\mathrm{Ba}$ for K) keeping $n_{c} \sim 0.4<n<1$, the ground state of the model is a Luttinger liquid with dominant charge density wave correlations at large distances, with wave vector $2 k_{F}=\pi n$. Experimentally, the ground state of $\mathrm{Ba}_{1-x} \mathrm{~K}_{x} \mathrm{BiO}_{3}$ has different charge density wave orderings for $0 \leq x<\sim 0.4(1 \geq n>\sim 0.6)$. As $n$ is further lowered (corresponding to increasing $x=1-n$ ), the model enters a region with dominant superconducting correlations at large distances, which has a corresponding superconfucting phase in $\mathrm{Ba}_{1-x} \mathrm{~K}_{x} \mathrm{BiO}_{3}$ (for $\sim 0.4<x<0.5$ ) U. Above $x=0.5$ the experimental system cannot be formed, since the solubility limit of $\mathrm{K}$ atoms is exceeded. This picture is also consistent with mean-field calculations in the three-dimensional model for different parameters 10 .

\section{ACKNOWLEDGMENTS}

We are partially supported by CONICET. This work was sponsored by PICT 03-00121-02153 of ANPCyT and PIP 4952/96 of CONICET.

${ }^{1}$ A.W. Sleight, J.L. Gillson, and P.E. Bierstadt, Solid State Commun. 17, 27 (1975).

${ }^{2}$ L.F. Mattheiss, E.M. Gyorgy, and D.W. Johnson, Jr., Phys. Rev. B 37, 3745 (1988); R.J. Cava, B. Battlog, J.J. Krajewski, R. Farrow, L.W. Rupp Jr., A.E. White, K. Short, W.F. Peck, and T. Kometani, Nature (London) 332, 814 (1988).

${ }^{3}$ B.Battlog, R.J. Cava, L.W. Rupp Jr., A.M. Mujsce, J.J. Krajewski, J.P. Remeika, W.F. Peck Jr., A.S. Cooper, and G.P. Espinosa, Phys. Rev. Lett 61, 1670 (1988). 
${ }^{4}$ C.K. Loong, P. Vashishta, R.K. Kalia, M.H. Degani, D.L. Price, J.D. Jorgensen, D.G. Hinks, B. Dabrowski, A.W. Mitchell, D.R. Richards, and Y. Zheng, Phys. Rev. Lett. 62, 2628 (1989).

${ }^{5}$ D.E. Cox and A.W. Sleight, Acta Crystallogr. B 35, 1 (1979).

${ }^{6}$ C. Chaillout, A. Santoro, J.P. Remeika, A.S. Cooper, G.P. Espinosa, and M. Marezio, Solid State Commun. 65, 1363 (1988).

${ }^{7}$ S. Pei, J.D. Jorgensen, B. Dabrowski, D.G. Hinks, D.R. Richards, A.W. Mitchell, J.M. Newsam, S.K. Sinha, D. Vaknin, and A.J. Jacobson, Phys. Rev. B 41, 4126 (1990).

8 M.D. Núñez Regueiro and A.A. Aligia, Phys. Rev. Lett. 61, 1889 (1988); J. Bala and A.M. Oleś, Phys. Rev. B 47, 515 (1993) ;A.A. Aligia, M.D. Núñez Regueiro, and E.R. Gagliano, ibid 40, 4405 (1989); A.A. Aligia and M. Baliña, ibid 47, 14380 (1993).

9 J.O. Sofo, A.A. Aligia, and M.D. Núñez Regueiro, Phys. Rev. B 39, 9701 (1989); 40, 6955 (1989).

${ }^{10}$ C.M. Varma, Phys. Rev. Lett. 61, 2713 (1988).

${ }^{11}$ D. Nguyen Manh, D. Mayou and F. Cyrot-Lackmann, Solid State Commun. 79, 723 (1991).

12 T.M. Rice and L. Sneddon, Phys. Rev. Lett. 47, 689 (1981).

${ }^{13}$ R. Micnas, J. Ranninger and S. Robaszkiewicz, Rev. Mod. Phys. 62, 113 (1990); references therein.

14 S. Salem-Sugui, Jr., E.E. Alp, S.M. Mini, M. Ramanathan, J.C. Campuzano, G. Jennings, M. Faiz, S. Pei, B. Dabrowski, Y. Zheng, D.R. Richards, and D.G. Hinks, Phys. Rev. B 43, 5511 (1991).

15 S.M. Heald, D. Di Marzio, M. Croft, M.S. Hedge, S. Li, and M. Greenblatt, Phys. Rev. B 40, 8828 (1989).

${ }^{16}$ S. Uchida, S. Tajima, A. Masaki, S. Sugai, K. Kitazawa, and S. Tanaka, J. Phys. Soc. Jpn 54, 4395 (1985).

${ }^{17}$ M.E. Simon, A.A. Aligia, and E. Gagliano, Phys. Rev. B 56, 5637 (1997); references therein. H. Rosner, H. Eschrig, R. Hayn, S.-L. Drechsler, and J. Málek, ibid 56, 3402 (1997); references therein..

${ }^{18}$ F. Mila and X. Zotos, Europhys. Lett. 24, 133 (1993).

${ }^{19}$ K. Penc and F. Mila, Phys. Rev. B 49, 9670 (1994).

${ }^{20}$ K. Sano and Y. Ono, J. Phys. Soc. Jpn. 63, 1250 (1994).

${ }^{21}$ H.Q. Lin, E.R. Gagliano, D.K. Campbell, E.H. Fradkin, and J.E. Gubernatis, in "The Physics and the Mathematical Physics of the Hubbard Model ", edited by D. Baeriswyl et al. (Plenum, New York, 1995).

${ }^{22}$ A.A. Aligia, Europhys. Lett. 45, 411 (1999).

${ }^{23}$ R.T. Clay, A.W. Sandvik, and D.K. Campbell, Phys. Rev. B 59, 4665 (1999).

${ }^{24}$ M. Nakamura, cond-mat/9909277.

25 J. Voit, Phys. Rev. Lett. 64323 (1990).

${ }^{26}$ J.W. Cannon and E. Fradkin, Phys. Rev. B 41, 9435 (1990).

27 J. Voit, Phys. Rev. B 45, 4027 (1992).

${ }^{28}$ A.A. Aligia and L. Arrachea, Phys. Rev. B in press (BG7464).

29 A.A. Aligia, Phys. Rev. B 47, 15308 (1993).

${ }^{30}$ C.D. Batista, F. Lema, and A.A. Aligia, Phys. Rev. B 52, 6223 (1995).

${ }^{31}$ The correlation exponent $K_{\rho}$ refers to the original model Eq. (1), and not to the spinless models. In general, the relations defining $K_{\rho}$ depend on the number of branches around the Fermi level [see for example G. Santoro, N. Manini, A. Parola, and E. Tosatti, Phys. Rev. B 53, 828 (1996)].

32 C.N. Yang and C.P. Yang, Phys. Rev. 150, 321 (1996); 150, 327 (1996).

${ }^{33}$ M. Fowler and M.W. Puga, Phys. Rev. B 18, 421 (1978).

${ }^{34}$ B. Sriram Shastry and B. Sutherland, Phys. Rev. Lett. 65, 243 (1990).

35 T. Giamarchi and A.M. Tsvelik, Phys. Rev. B 59, 11398 (1999); references therein.

${ }^{36}$ F. Lema, C.D. Batista, and A.A. Aligia, Physica C 259, 287 (1996). 